\title{
Symmetry-derived selection rules for plasmon-enhanced Raman scattering
}

\author{
Ado Jorio ${ }^{1}$, Niclas S. Mueller ${ }^{2}$, Stephanie Reich ${ }^{2}$ \\ ${ }^{1}$ Departamento de Fúsica, Universidade Federal de Minas Gerais, \\ Belo Horizonte, MG 30123-970, Brazil. \\ ${ }^{2}$ Department of Physics, Freie Universität Berlin, \\ Arnimallee 14, D-14195 Berlin, Germany.
}

(Dated: Submitted on March 20, 2017)

\begin{abstract}
We show how to obtain the symmetry-imposed selection rules for plasmonic enhancement in surface- (SERS) and tip-enhanced Raman scattering (TERS). Plasmon-enhanced light scattering is described as a higher-order Raman process, which introduces a series of Hamiltonians representing the interaction between light, plasmons, electrons, and phonons. Using group theory we derive the active representations for point group symmetries of exemplary plasmonic nanostructures. The phonon representations that are enhanced by SERS and TERS are then found as induced representations for the symmetry group of the molecule or another Raman probe. The selection rules are discussed for graphene that is coupled to a nanodisk dimer as an example for SERS and coupled to a tip as a TERS example. The phonon eigenmodes that are enhanced depend on the symmetry breaking when combining the plasmonic structures with graphene. We show that the most prominent optical phonon modes $\left(E_{2 g}\right.$ and $\left.A_{1 g}\right)$ are allowed in all scattering configurations when using a nanodimer as a plasmonic hotspot. We predict the activation of the silent $B_{2 g}$ as well as infrared-active $A_{2 u}$ and $E_{1 u}$ modes in SERS for crossed configurations of the incoming and scattered light. There is a systematic difference between spatially coherent and incoherent plasmon-enhanced Raman scattering, which is responsible for a dependence of TERS on the phonon coherence length.
\end{abstract}




\section{INTRODUCTION}

Surface-enhanced Raman scattering (SERS) describes the giant increase in the Raman scattering cross section close to metal nanostructures and rough metal surfaces. ${ }^{1-4}$ Under special conditions the SERS intensity is strong enough to provide single-molecule sensitivity. ${ }^{5,6}$ While originally rough metal surfaces were used as sources of SERS enhancement, controlled plasmonic nanostructures are nowadays often employed as SERS hotspots. ${ }^{7}$ Related to SERS is tip-enhanced Raman scattering (TERS), where a plasmonic metal tip is scanned over a Raman probe. ${ }^{3,8}$ TERS provides a high level of control over the strength and position of plasmonic enhancement. It allows scanning the surface or imaging nanostructures with a plasmonic near field. ${ }^{9}$ Its ultimate resolution was recently demonstrated by intramolecular TERS imaging. ${ }^{10}$ The dominant enhancement mechanism that leads to SERS and TERS is the interaction of the Raman probe with the plasmonic near field of a metal nanostructure. ${ }^{3,4,11}$ We therefore adopt the term plasmon-enhanced Raman scattering (PERS) to address the effect generally, including SERS and TERS. There are many efforts to describe PERS theoretically ${ }^{12-16}$ including recent innovative approaches such as molecular cavity optomechanics ${ }^{17,18}$ and the description as a higher-order Raman process ${ }^{16}$.

The study of linear and non-linear optical processes in molecules and solids greatly benefits from analyzing the symmetry-imposed selection rules. ${ }^{19-21}$ In Raman scattering recording a mode intensity as a function of polarization of the incoming and inelastically scattered light allows assigning eigenmodes to the experimentally recorded spectra. ${ }^{20,22}$ Group theory and symmetry arguments were used to obtain plasmon eigenvectors of metallic nanostructures within a hybridization model. ${ }^{23-25}$ It was also shown that elastic light scattering and absorption are well explained by treating plasmonic trimmers as artificial molecules with $D_{\infty h}$ point group symmetry for a linear chain and $D_{3 h}$ for a triangular arrangement. ${ }^{23}$ PERS, however, is not generally analyzed by group theory, although attempts were made for such an analysis in specific examples. ${ }^{13,26}$ Results of electromagnetic calculations of TERS intensities in graphene were also discussed in view of the symmetry of the setup. ${ }^{27}$ Part of the problem is that plasmonic enhancement in Raman scattering is often simplified as an increase in local field intensity neglecting the presence of distinct selection rules and the symmetry of the plasmonic near field. Approaches for describing SERS within the microscopic theory of Raman scattering found it challenging to include the transformation properties of the 
interaction Hamiltonians that governed photon-electron coupling via plasmon excitation. The experimental observation of infrared-active vibrations in the SERS spectra were, therefore, analyzed in terms of the selection rules for the Raman effect combined with symmetry breaking on a metallic surface. ${ }^{13}$

Here we show that the description of PERS as a higher-order Raman process provides a natural way to establish the selection rules for PERS. We introduce a methodology for treating plasmonic enhancement within group theory. The selection rules are found for distinct sets of point groups of the plasmonic nanostructure and the Raman probe. We first derive PERS-active representations for a given plasmonic system, i.e., for a given point group, considering various experimental configurations. Secondly, we consider how a Raman probe, which can be a molecule or a nanostructure, will have specific vibrational modes enhanced by the plasmonic system, thus deriving the PERS selection rules for the specific Raman probe-plasmonic structure coupled system. As an example, we demonstrate such an analysis for graphene as a two-dimensional system to study the fundamentals of SERS and TERS. The agreement between our symmetry-based conclusions and previous theoretical calculation $^{27,28}$ and experimental observations ${ }^{29,30}$ gives strong support for the description of PERS as a higher-order Raman process. ${ }^{16}$

The paper is organized as follows: Section II presents the theoretical tools used in the analysis and simulations of the study. Section III introduces the general framework for obtaining the selection rules based on group theory. The PERS-active representations and optical selection rules of a nanodisk dimer and other nanostructures are obtained in Sect. IV. Section V shows how to derive the SERS and TERS selection rules for the exemplary case of graphene. Section VI summarizes the main findings.

\section{METHODS}

The majority of arguments presented in this paper are based on group theory and the reader is referred to standard textbooks. ${ }^{19-21}$ The analysis requires straightforward (but somewhat tedious) manipulations like finding the irreducible representations of direct products and setting up correlation tables. Besides textbooks that collected such information, e.g., Wilson et. al. ${ }^{20}$, we found two online resources particularly helpful in facilitating the analysis: The Bilbao Crystallographic Server ${ }^{31,32}$ and the tables for point groups compiled 
by Gernot Katzer. ${ }^{33}$ For the $D_{2 h}$ point group we use $z$ as the basis function for $B_{1 u}, y$ for $B_{2 u}$, and $x$ for $B_{3 u}$, which is the convention most commonly found in the group-theory literature. For the other groups, the major symmetry axis is along $z$.

We numerically calculated the plasmonic properties of a dimer that was constructed from two silver nanodisks with a diameter $d=25 \mathrm{~nm}$ and a separation of $5 \mathrm{~nm}$ between the disks. The dielectric constant of the environment was set to $\epsilon=2.25$. This choice ensures that the eigenenergies are well separated in energy. The eigenmodes and eigenenergies were calculated using the eigenmode solver of the MNPBEM Matlab package that is based on the boundary-elements method. ${ }^{34,35}$ The absorption cross section was found within the quasi-static approximation, which very well describes $\mathrm{Ag}$ nanostructures with dimensions $(\approx 50 \mathrm{~nm})$ that are much smaller than the wavelength of the light $(\approx 500 \mathrm{~nm})$.

\section{GENERAL GROUP THEORY FRAMEWORK OF PERS}

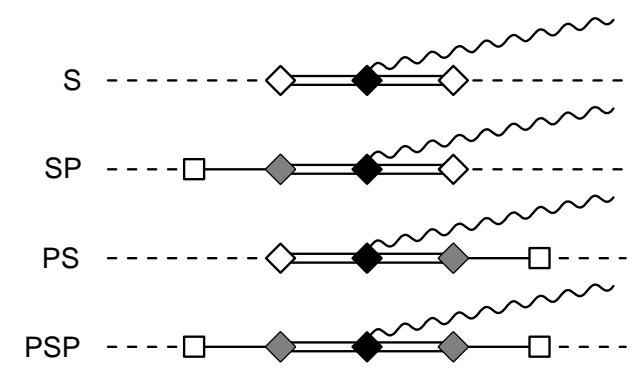

FIG. 1. Feynman diagrams for the PERS effect as a higher-order scattering event. Dashed line indicates a photon, single-solid line a plasmon, double-solid line an electron-hole pair and wavysolid line a phonon. $\square$, white, gray and black $\diamond$ indicate photon-plasmon (pt-pl), photon-electron (pt-el), plasmon-electron (pl-el) and electron-phonon (el-pn) interactions, respectively. $\mathbf{S}$ is the usual Raman scattering Stokes process, where light interacts only with the sample. SP and PS are processes where the interaction of the incoming and outgoing light, respectively, is mediated by the plasmonic structure. PSP is a process where both incoming and outgoing light interactions are mediated by the plasmon [the nomenclature used here follows Ref. 3, by replacing $\mathbf{T}$ for tip (in TERS) by $\mathbf{P}$ for plasmon, the order of $\mathbf{P}$ or $\mathbf{S}$ following the operator logic, i.e., events happen from the right to the left]. 
We adopt the microscopic theory of Raman scattering and treat PERS as higher-order Raman scattering. ${ }^{16}$ The PERS process is then described by a series of possible interaction processes between sample and plasmonic structure; 3,16 the most fundamental processes are depicted as Feynman diagrams in Fig. 1. The first diagram $\mathbf{S}$ is the usual Raman scattering Stokes process, where $\mathbf{S}$ stands for "sample", and light interacts only with the sample itself. This process does not make use of the plasmonic enhancement and its selection rules are well established. ${ }^{22,36}$ In the SP and PS processes the interaction of the incoming and outgoing light, respectively, are mediated by the plasmonic $(\mathbf{P})$ structure, and the plasmon is responsible for exciting or de-exciting the sample. In the PSP process, both incoming and outgoing light interactions are mediated by the plasmon. In a PERS experiment all four processes will happen simultaneously (and also some more with different time-order, neglecting higher-order terms). Their relative contribution will depend on the energetic separation between the energy of the plasmonic excitation, the laser energy and the Raman probe phonon energy, which defines the scattered light energy. It will also depend on the relative strength of the coupling between plasmon-photon and plasmon-electron compared to electron-photon coupling. ${ }^{16}$ In SERS experiments with strongest enhancement $\left(10^{8}-10^{10}\right.$ increase in scattering cross section) PSP processes are by far dominant. However, for weaker SERS hotspots, for extended (two-dimensional) nanostructures, for less favorable orientation of the Raman probe to the direction of the near fields, and for certain external polarization conditions, the SP and PS processes may become important.

The Feynman diagrams in Fig. 1 are evaluated into scattering amplitudes using Fermi's golden rule. For example, PSP yields ${ }^{16,22}$

$K_{\mathbf{P S P}}^{p n}\left(\omega_{L}\right)=\frac{M_{\mathbf{P S P}}^{p n}}{\left(E_{L}-E_{p l}^{\prime}-i \gamma_{p l}\right)\left(E_{L}-E_{e l}^{a}-i \gamma_{e l}\right)\left(E_{L}-\hbar \omega_{p n}-E_{e l}^{b}-i \gamma_{e l}\right)\left(E_{L}-\hbar \omega_{p n}-E_{p l}^{\prime \prime}-i \gamma_{p l}\right)}$,

where $E_{L}$ is the laser energy and $\hbar \omega_{p n}$ the phonon energy. $E_{e l}^{a}$ and $E_{e l}^{b}$ are excited electronic states of the Raman scatterer with inverse lifetime $\gamma_{e l}$ (taken to be the same for simplicity). $E_{p l}^{\prime}$ and $E_{p l}^{\prime \prime}$ are energies of plasmonic eigenstates of the nanostructure with inverse lifetime $\gamma_{p l}$ (identical for simplicity). The denominator in Eq. (1) gives rise to the characteristic resonances in the Raman cross section if the laser energy matches an electronic excitation of the Raman probe (usual resonant Raman scattering) or the plasmonic nanostructure 
(PERS), see Ref. 16 for a discussion.

To derive the symmetry-imposed selection rules of Eq. (1) we need to evaluate the combined matrix element $M_{\mathbf{P S P}}^{p n}$. It has the form ${ }^{16,22}$

$$
\begin{aligned}
M_{\mathbf{P S P}}^{p n}= & \left\langle\psi_{\Gamma_{e l}^{f} \otimes \Gamma_{p l}^{f}}\left|\mathcal{H}_{\mathrm{pt}-\mathrm{pl}}\right| \psi_{\Gamma_{e l}^{f} \otimes \Gamma_{p l}^{\prime \prime}}\right\rangle \times \\
& \left\langle\psi_{\Gamma_{e l}^{f} \otimes \Gamma_{p l}^{\prime \prime}}\left|\mathcal{H}_{\mathrm{pl}-\mathrm{el}}\right| \psi_{\Gamma_{e l}^{b} \otimes \Gamma_{p l}^{i}}\right\rangle \times \\
& \left\langle\psi_{\Gamma_{e l}^{b} \otimes \Gamma_{p l}^{i}}\left|\mathcal{H}_{\mathrm{el}-\mathrm{pn}}\right| \psi_{\Gamma_{e l}^{a} \otimes \Gamma_{p l}^{i}}\right\rangle \times \\
& \left\langle\psi_{\Gamma_{e l}^{a} \otimes \Gamma_{p l}^{i}}\left|\mathcal{H}_{\mathrm{pl}-\mathrm{el}}\right| \psi_{\Gamma_{e l}^{i} \otimes \Gamma_{p l}^{\prime}}\right\rangle \times \\
& \left\langle\psi_{\Gamma_{e l}^{i} \otimes \Gamma_{p l}^{\prime}}\left|\mathcal{H}_{\mathrm{pt}-\mathrm{pl}}\right| \psi_{\Gamma_{e l}^{i} \otimes \Gamma_{p l}^{i}}\right\rangle,
\end{aligned}
$$

where the symmetry of the state $\left|\psi_{\Gamma_{e l}^{\beta} \otimes \Gamma_{p l}^{\alpha}}\right\rangle$ is defined by the symmetries of the plasmonic system $\left(\Gamma_{p l}^{\alpha}\right)$ and of the sample (molecule or nanostructure) electronic state $\left(\Gamma_{e l}^{\beta}\right)$. The selection rules for the PSP process are built from a sequence of considerations which follows the perturbation Hamiltonian of Eq. (2):

1. $\left|\psi_{\Gamma_{e l}^{i} \otimes \Gamma_{p l}^{i}}\right\rangle$ describes the system in its initial state $(i)$, which is composed of the plasmonic structure and the electronic structure of the sample in their initial symmetry state $\Gamma_{p l}^{i}$ and $\Gamma_{e l}^{i}$, respectively. At the initial state, both the electronic and plasmonic systems are considered to be in the ground state. From the group-theory point of view, they are represented by totally symmetric irreducible representations $\left(\Gamma_{1}\right)$.

2. $\mathcal{H}_{\mathrm{pt}-\mathrm{pl}}$ is the photon-plasmon interaction operator, which creates a plasmon and annihilates a photon, thus changing the plasmonic system symmetry from $\Gamma_{p l}^{i}$ to $\Gamma_{p l}^{\prime}$. $\mathcal{H}_{\mathrm{pt}-\mathrm{pl}}$ has the form $\mathbf{p} \cdot \mathbf{E}$, where $\mathbf{p}$ stands for the plasmonic dipole moment, and $\mathbf{E}$ is the light electric field. A broadly used approximation is considering that light has a large wavelength when compared to the nanostructures considered here, then the dipole approximation is valid and the $\mathcal{H}_{\mathrm{pt}-\mathrm{pl}}$ operator exhibits the symmetry of a vector. Therefore, since the initial state is totally symmetric, the matrix element $\left\langle\psi_{\Gamma_{e l}^{i} \otimes \Gamma_{p l}^{\prime}}\left|\mathcal{H}_{\mathrm{pt}-\mathrm{pl}}\right| \psi_{\Gamma_{e l}^{i} \otimes \Gamma_{p l}^{i}}\right\rangle$ is non-zero when the irreducible representation $\Gamma_{p l}^{\prime}=\Gamma_{v e c}$, where $\Gamma_{v e c}$ is the irreducible representation of a vector (basis functions $x, y$, and $z$ ).

3. $\mathcal{H}_{\mathrm{pl}-\mathrm{el}}$ is the plasmon-electron interaction operator, which annihilates a plasmon by exciting an electron-hole pair. The plasmonic system goes back to the totally symmetric state, while the Raman probe is changed from the totally symmetric $\Gamma_{e l}^{i}$ to $\Gamma_{e l}^{a}$. 
The matrix element $\left\langle\psi_{\Gamma_{e l}^{a} \otimes \Gamma_{p l}^{i}}\left|\mathcal{H}_{\mathrm{pl}-\mathrm{el}}\right| \psi_{\Gamma_{e l}^{i} \otimes \Gamma_{p l}^{\prime}}\right\rangle$ is non-zero if $\Gamma_{e l}^{a} \subset \Gamma^{\mathcal{H}_{\mathrm{pl}-\mathrm{el}}} \otimes \Gamma_{v e c}$, and the result depends on the symmetry of the $\mathcal{H}_{\mathrm{pl}-\mathrm{el}}$ operator. Similar to the case of $\mathcal{H}_{\mathrm{pt}-\mathrm{pl}}$, $\mathcal{H}_{\mathrm{pl}-\mathrm{el}}$ has the form $\mathbf{p} \cdot \mathbf{E}$, where $\mathbf{p}$ is the electronic dipole moment of the Raman probe, and $\mathbf{E}$ is the electric field generated by the plasmon.

Two different perspectives onto the symmetry properties of $\mathbf{E}$ are implicitly or explicitly adopted in the scientific literature, ${ }^{26-28}$ a spatially incoherent (local) and a spatially coherent (global) description. The incoherent approach considers the near field of the plasmon in a given point. It describes $\mathbf{E}$ as a dipole with symmetry $\Gamma_{\text {vec }}$ or in a multipolar expansion $\Gamma^{\mathcal{H}_{\mathrm{pl}-\mathrm{el}}}=\Gamma_{\text {vec }} \oplus \Gamma_{\text {quad }} \oplus \ldots$, where quad stands for quadrupole. The spatially incoherent description is appropriate for strictly localized Raman scatterers, for example, a molecule on the tip of an atomic-force microscope (AFM), ${ }^{37}$ an ultra-sharp silicon tip, ${ }^{38}$ and, in general, molecular probes. The spatially coherent approach applies for extended samples such as two-dimensional materials interacting with plasmonic structures. ${ }^{9,27,29,30,39,40}$ In this case, PERS amplitudes from different areas of the near field interfere. The symmetry of $\mathbf{E}$ is given by the transformation properties of the entire near-field distribution or the plasmon eigenmode. This implies that we need to evaluate the plasmonic eigenmodes of the nanostructure. ${ }^{23,25}$

The symmetry of the perturbation $\Gamma^{\mathcal{H}_{\mathrm{pl}-\mathrm{el}}}$ will be considered case by case. Up to this point the selection rule can be given by $\Gamma_{e l}^{a} \subset \Gamma^{\mathcal{H}_{\mathrm{pl}-\mathrm{el}}} \otimes \Gamma_{v e c}$.

4. $\mathcal{H}_{\mathrm{el}-\mathrm{pn}}$ is the electron-phonon interaction operator that creates/annihilates a phonon (Stokes/anti-Stokes process) by changing the electronic system from symmetry $\Gamma_{e l}^{a}$ to $\Gamma_{e l}^{b}$. The matrix element $\left\langle\psi_{\Gamma_{e l}^{b} \otimes \Gamma_{p l}^{i}}\left|\mathcal{H}_{\mathrm{el}-\mathrm{pn}}\right| \psi_{\Gamma_{e l}^{a} \otimes \Gamma_{p l}^{i}}\right\rangle$ will be non-zero if $\Gamma_{e l}^{b} \subset \Gamma^{\mathcal{H}_{\text {el-pn }}} \otimes \Gamma_{e l}^{a}$. $\Gamma^{\mathcal{H}_{\mathrm{el}-\mathrm{pn}}}$ exhibits the symmetry of the phonon $\left(\Gamma_{p n}\right)$ yielding $\Gamma_{e l}^{b} \subset \Gamma_{p n} \otimes \Gamma^{\mathcal{H}_{\mathrm{pl}-\mathrm{el}}} \otimes \Gamma_{\text {vec }}$.

5. In the sequence, the operator $\mathcal{H}_{\mathrm{pl}-\mathrm{el}}$ creates a plasmon of symmetry $\Gamma_{p l}^{\prime \prime}$ by changing the electronic system from $\Gamma_{e l}^{b}$ to $\Gamma_{e l}^{f}$. The sample's electronic system will be already in the final state, therefore belonging to the totally symmetric $\left(\Gamma_{1}\right)$ irreducible representation (i.e., $\Gamma_{e l}^{f}=\Gamma_{1}$ ). The matrix element $\left\langle\psi_{\Gamma_{e l}^{f} \otimes \Gamma_{p l}^{\prime \prime}}\left|\mathcal{H}_{\mathrm{pl}-\mathrm{el}}\right| \psi_{\Gamma_{e l}^{b} \otimes \Gamma_{p l}^{i}}\right\rangle$ will be non-zero if $\Gamma_{p l}^{\prime \prime} \subset$ $\Gamma^{\mathcal{H}_{\mathrm{pl}-\mathrm{el}}} \otimes \Gamma_{e l}^{b} \otimes \Gamma_{p l}^{i}$, which means that $\Gamma_{p l}^{\prime \prime} \subset \Gamma^{\mathcal{H}_{\mathrm{pl}-\mathrm{el}}} \otimes \Gamma_{p n} \otimes \Gamma^{\mathcal{H}_{\mathrm{pl}-\mathrm{el}}} \otimes \Gamma_{v e c}$.

6. At the end of the PSP process, the system will have to go back to the ground state, which happens via $\mathcal{H}_{\mathrm{pt}-\mathrm{pl}}$ destroying the plasmon quantum and generating a pho- 
ton, thus taking the plasmonic system back to the totally symmetric state. Therefore, considering $\mathcal{H}_{\mathrm{pt}-\mathrm{pl}}$ is represented by $\Gamma_{v e c}$, the last matrix element of Eq. (2), $\left\langle\psi_{\Gamma_{e l}^{f} \otimes \Gamma_{p l}^{f}}\left|\mathcal{H}_{\mathrm{pt}-\mathrm{pl}}\right| \psi_{\Gamma_{e l}^{f} \otimes \Gamma_{p l}^{\prime \prime}}\right\rangle$ is non-zero if

$$
\Gamma_{v e c} \otimes \Gamma^{\mathcal{H}_{\mathrm{pl}-\mathrm{el}}} \otimes \Gamma_{p n} \otimes \Gamma^{\mathcal{H}_{\mathrm{pl}-\mathrm{el}}} \otimes \Gamma_{v e c} \supset \Gamma_{1}
$$

The selection rules for the $\mathbf{S P}$ and PS processes are obtained by removing the respective $\Gamma^{\mathcal{H}_{\mathrm{pt}-\mathrm{pl}}}=\Gamma_{v e c}$ outer-term in Eq. (3), and assuming the dipole approximation for the photonelectron interaction operator $\mathcal{H}_{\mathrm{pt}-\mathrm{el}}$, i.e., the consecutive $\Gamma^{\mathcal{H}_{\mathrm{pl}-\mathrm{el}}}$ symmetry in Eq. (3) will be replaced by $\Gamma^{\mathcal{H}_{\mathrm{pt}-\mathrm{el}}}=\Gamma_{v e c}$.

In summary, we obtained the following selection rules for the processes in Fig. 1

$$
\begin{gathered}
\mathbf{S}:\left(\Gamma_{v e c} \otimes \Gamma_{v e c}\right) \subset \Gamma_{p n} \\
\text { SP }, \mathbf{P S}:\left(\Gamma_{v e c} \otimes \Gamma^{\mathcal{H}_{p l-e l}} \otimes \Gamma_{v e c}\right) \subset \Gamma_{p n} \\
\mathbf{P S P}:\left(\Gamma_{v e c} \otimes \Gamma^{\mathcal{H}_{p l-e l}} \otimes \Gamma^{\mathcal{H}_{p l-e l}} \otimes \Gamma_{v e c}\right) \subset \Gamma_{p n} .
\end{gathered}
$$

At this point one may proceed by finding the point group of the plasmonic nanostructure plus the Raman probe and use it to evaluate Eq. (4). However, the point group symmetry of the combined system will, in general, be very low and depend critically on the exact position and orientation of its components. Even small changes will require completely reevaluating the selection rules. Therefore, it is interesting to first consider the plasmonic nanostructure, its symmetry properties and selection rules according to Eq.(4), thus finding the PERS-active representations within the plasmonic nanostructure symmetry. Then, one can find the irreducible representations of the Raman probe phonons that can be enhanced by the plasmonic system ("PERS-active phonons"), using a correlation-table analysis. ${ }^{19-21}$ With such a procedure one is able to make an easy connection between the PERS-active phonons and the phonons of the Raman probe independently of the plasmonic structure.

Next we will find the symmetry properties and selection rules of a given plasmonic structure according to Eqs.(4), thus finding the PERS-active representations of that specific plasmonic nanostructure (see Section IV). Then, we will seek the irreducible representation of a given Raman probe induced by the plasmonic system via a correlation-table analysis between the symmetry groups of the two systems (Raman probe and plasmonic nanostructure), thus finding the PERS-active phonons (see Section V). ${ }^{19-21}$ 


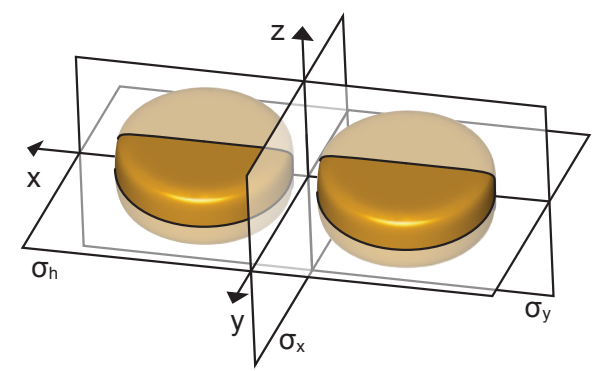

FIG. 2. Schematics of the $D_{2 h}$ dimer structure with some of the symmetry elements indicated. The symmetry operations are the identity $E, C_{2}$ axis along the $x, y$ and $z$ directions, the inversion symmetry $i$, and mirror planes $\sigma$ perpendicular to the $x, y$ and $z\left(\sigma_{h}\right)$ axes. The invariant point is at the dimer center (cartesian coordinate origin), where the inversion symmetry is located.

\section{PERS-ACTIVE REPRESENTATIONS FOR SPECIFIC PLASMONIC NANOS- TRUCTURES}

In this section we derive the PERS-active representations for a given symmetry of the plasmonic nanostructure. We will evaluate a nanodisk dimer in detail, considering spatially coherent and incoherent PERS processes. From the symmetry standpoint, the results are consistent with other dimer structures of interest, such as the bowtie nanoantennae. ${ }^{41}$ Furthermore, the results for other nanostructures will be presented in tabular format.

\section{A. Spatially incoherent PERS processes}

In the approximation of incoherent PERS, scattering intensities from different areas will add up without interferences. The symmetry $\Gamma^{\mathcal{H}_{p l-e l}}=\Gamma_{v e c}$ when restricting to the dipole approximation (the extension to higher-order multipoles is straightforward, but cumbersome). We now consider a plasmonic nanodisk dimer, see Fig. 2. It has a point group of $D_{2 h}$, the symmetry operations are indicated in Fig. 2. For this structure and the dipole approximation Eqs. (4b) and (4c) evaluate to

$$
\begin{aligned}
& \Gamma_{\mathbf{S P}, \mathbf{P S}}^{\mathrm{incoh}}=A_{u} \oplus B_{1 u} \oplus B_{2 u} \oplus B_{3 u} \\
& \Gamma_{\mathbf{P S P}}^{\mathrm{incoh}}=A_{g} \oplus B_{1 g} \oplus B_{2 g} \oplus B_{3 g} .
\end{aligned}
$$

The incoherent PSP process for a $D_{2 h}$ point group plasmonic structure has selection rules that are identical to the conventional (non-PERS) Raman selection rules for a $D_{2 h}$ 
TABLE I. Irreducible representations (IR) that are PERS-active within the symmetry of specific plasmonic nanostructures, for spatially incoherent PSP- and SP, PS-type processes, compare Fig. 1. The second column lists example nanostructures that belong to the point groups. "tip" stands for a TERS tip (cone structure), and "tip and image" stands for TERS in the gap mode geometry (tip normal to a metallic plane). For the (semi)infinite group $\left(C_{\infty v}\right) D_{\infty h}$ we listed the two usual irreducible representation nomenclatures (e.g. for $D_{\infty h}, \Sigma_{g}^{+}=A_{1 g}$ ).

\begin{tabular}{llll}
\hline \hline point group & example & PSP-active IR & SP, PS-active IR \\
\hline$D_{2 h}$ & disk dimer, bowtie & $A_{g}, B_{1 g}, B_{2 g}, B_{3 g}$ & $A_{u}, B_{1 u}, B_{2 u}, B_{3 u}$ \\
$D_{3 h}$ & trimer & $A_{1}^{\prime}, A_{1}^{\prime \prime}, A_{2}^{\prime \prime}, E^{\prime}, E^{\prime \prime}$ & $A_{1}^{\prime}, A_{2}^{\prime \prime}, E^{\prime}, E^{\prime \prime}$ \\
$D_{4 h}$ & square & $A_{1 g}, A_{2 g}, B_{1 g}, B_{2 g}, E_{g}$ & $A_{2 u}, B_{1 u}, B_{2 u}, E_{u}$ \\
$D_{6 h}$ & hexagon & $A_{1 g}, B_{1 g}, B_{2 g}, E_{1 g}, E_{2 g}$ & $A_{2 u}, B_{1 u}, B_{2 u}, E_{1 u}, E_{2 u}$ \\
$D_{\infty h}$ & sphere dimer, tip and image & $\Sigma_{g}^{+}, \Pi_{g}, \Delta_{g}, \Phi_{g}, \Gamma_{g}$ & $\Sigma_{u}^{+}, \Pi_{u}, \Delta_{u}, \Phi_{u}$ \\
& & $A_{1 g}, E_{1 g}, E_{2 g}, E_{3 g}, E_{4 g}$ & $A_{2 u}, E_{1 u}, E_{2 u}, E_{3 u}$ \\
\hline$C_{2 v}$ & asymmetric dimer & $A_{1}, A_{2}, B_{1}, B_{2}$ & $A_{1}, A_{2}, B_{1}, B_{2}$ \\
$C_{3 v}$ & & $A_{1}, A_{2}, E$ & $A_{1}, A_{2}, E$ \\
$C_{4 v}$ & & $A_{1}, A_{2}, B_{1}, B_{2}, E$ & $A_{1}, B_{1}, B_{2}, E$ \\
$C_{\infty v}$ & $\Sigma^{+}, \Pi, \Delta, \Phi, \Gamma$ & $\Sigma^{+}, \Pi, \Delta, \Phi$ \\
& tip & $A_{1}, E_{1}, E_{2}, E_{3}, E_{4}$ & $A_{1}, E_{1}, E_{2}, E_{3}$ \\
\hline \hline
\end{tabular}

point group $\left(\Gamma_{\text {Raman }}^{D_{2 h}}=A_{g} \oplus B_{1 g} \oplus B_{2 g} \oplus B_{3 g}\right)$. However, we have not yet considered the Raman probe. The PERS-active phonons will still depend on the correlation between the Raman probe symmetry and the plasmon structure symmetry ( $D_{2 h}$ point group here), and they will be derived in Section $\mathrm{V}$ for graphene, which exhibits $D_{6 h}$ symmetry. In contrast SP, PS processes occur only for representations that are ungerade, see Table I. These representations would be hyper-Raman active and some infrared active in a $D_{2 h}$ point group Raman probe without plasmonic enhancement.

Table I summarizes the selection rules for other important point groups. In all point groups with inversion symmetry we find a clear parity separation between the irreducible representations that are active in the $\mathbf{P S P}$ versus $\mathbf{S P}, \mathbf{P S}$-type processes. $D_{\infty h}$ point group is representative of a SERS prototype plasmonic system, the sphere dimer, and also of 
a TERS system within the gap mode, where the substrate is a metallic surface that can be replaced by an identical tip using the method of image charges. $C_{\infty v}$ point group is representative for a regular TERS system.

\section{B. Spatially coherent PERS processes}

(a)

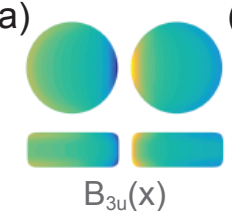

(d)

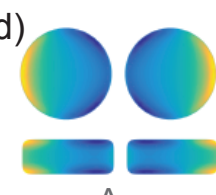

(g)

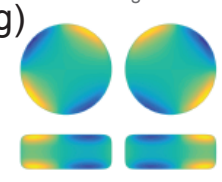

$\mathrm{B}_{2 \mathrm{u}}(\mathrm{y})$ (b)

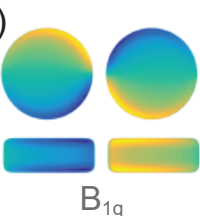

(e)

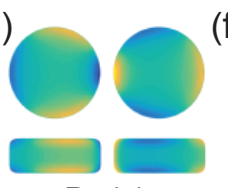

(h)

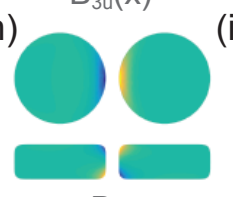

$\mathrm{B}_{2 \mathrm{~g}}$ (c)

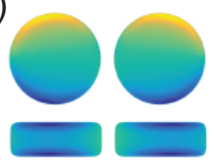

$\mathrm{B}_{2 \mathrm{u}}(\mathrm{y})$

(f)

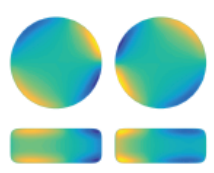

(i)

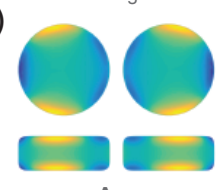

FIG. 3. Calculated charge density distributions for the plasmonic eigenmodes, along with the respective irreducible representations and the $x$ and $y$ basis functions (in parenthesis). For each panel, the charge distribution on the dimer is shown from the top and from the side.

Spatially coherent scattering appears to be dominant for extended, crystalline samples, because the dimension of the plasmonic near field is often comparable or even smaller than the phonon coherence length. ${ }^{27,29}$ Under these conditions we need to examine the symmetry properties of the plasmonic eigenmodes excited during the PERS process, which will define the symmetry of the perturbation $\Gamma^{\mathcal{H}_{p l-e l}}=\Gamma_{p l}$. Figures $3(\mathrm{a})-(\mathrm{i})$ show the calculated surfacecharge densities for the plasmonic eigenstates of the nanodisk dimer. The first nine modes are shown, ordered by increasing eigenenergy and labeled with their irreducible representations.

Plasmons belonging to the $\Gamma_{v e c}=B_{1 u} \oplus B_{2 u} \oplus B_{3 u}$ representations can be excited by light in the far-field regime (dipole approximation). $B_{1 u}$ is excited with $z$ polarized light, $B_{2 u}$ with $y$, and $B_{3 u}$ with $x$ polarization. Two $B_{3 u}$ plasmons are shown in Fig. 3(a) and (e) and two $B_{2 u}$ plasmons in Figs. 3(c) and (g). The eigenmodes in Figs. 3(a) and (c) are obtained by combining two dipole-like functions in the two disks oriented along $x$ and $y$, respectively. 
Figures $3(\mathrm{e})$ and $(\mathrm{g})$ are obtained from quadrupolar eigenmodes in each disk. $B_{1 u}$ modes appear at higher energies and are not shown in Fig. 3.
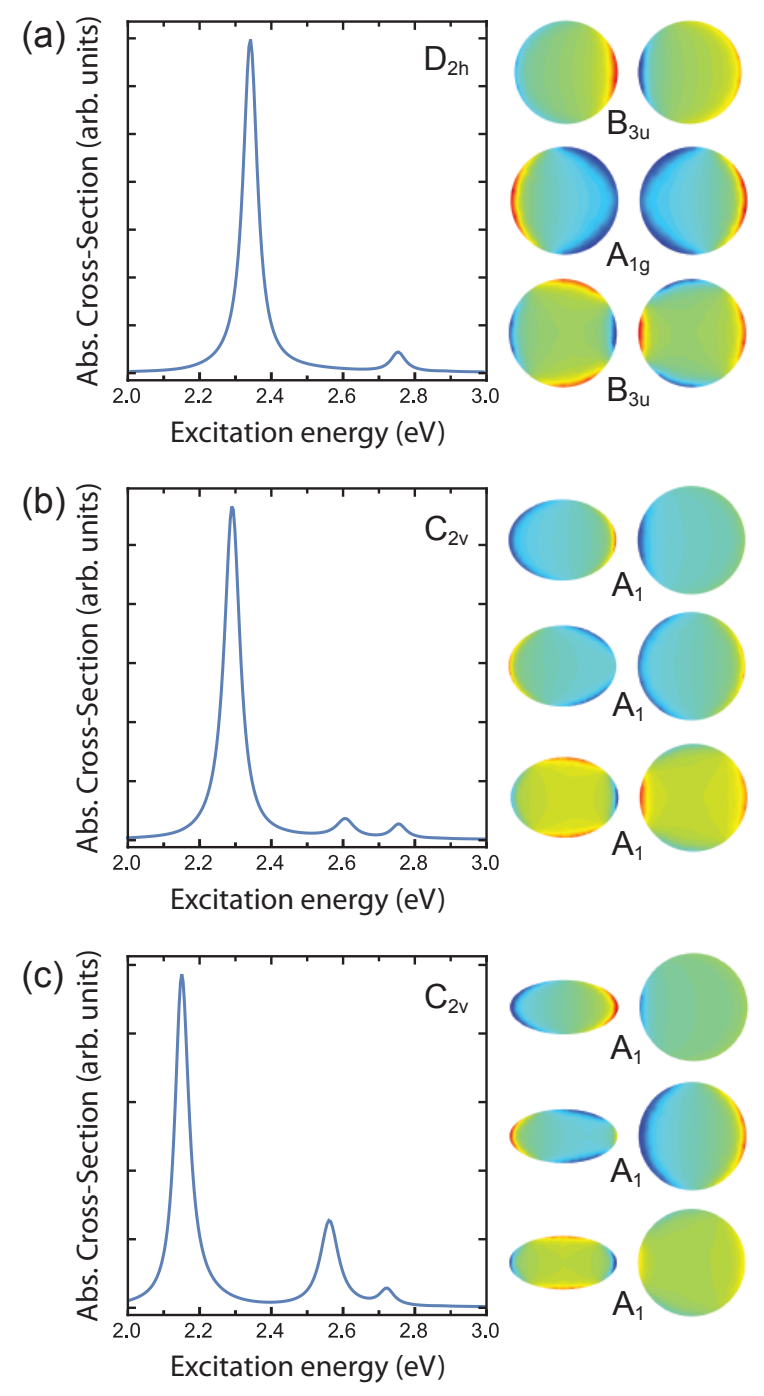

FIG. 4. Calculated optical absorption cross sections (left) with peaks originating from the three plasmon modes displayed on the right side of the plots. The quasi-static approximation is used with light polarization along the dimer axis. (a) Nanodisk dimer made of two perfectly circular disks $\left(D_{2 h}\right.$ symmetry). (b) and (c) are for dimers with increasing level of symmetry breaking by imposing an elliptical shape to one of the disks $\left(C_{2 v}\right.$ symmetry). The ratio between the long and the short axes are 100:75 in (b) and 100:50 in (c). The irreducible representations for the plasmons are indicated next to the panels in the right side of the plots.

Figure 4(a) shows the optical absorption from the dimer, for light polarized along the dimer axis ( $x$ axis, see Fig. 2$)$. The bright $B_{3 u}$ dipole-like plasmon mode [upper-right panel 
in Fig. 4(a)] appears at $2.34 \mathrm{eV}(529 \mathrm{~nm})$. It is one order of magnitude stronger than the quadrupole-derived $B_{3 u}$ mode [bottom-right panel in Fig. 4(a)] at $2.75 \mathrm{eV}(450 \mathrm{~nm})$. The $A_{1 g}$ symmetry plasmon [middle-right panel in Fig.4(a)] is symmetry-forbidden for far-field excitation; it is a dark mode. Figures 4(b) and (c) show the effect of breaking the dimer symmetry by imposing an elliptical shape on one of the disks. In this case, the system lowers its symmetry from $D_{2 h}$ to $C_{2 v}$ with the $C_{2}$ axis along $x$, loosing the inversion symmetry, the $\sigma^{x}$ mirror and the $C_{2}^{y}$ and $C_{2}^{z}$ axes. From the compatibility relations, the $B_{3 u}$ and $A_{1 g}$ irreducible representations go into the $A_{1}$ irreducible representation in $C_{2 v}\left(C_{2}\right.$ along $\left.x\right)$, which has $x$ as the basis functions. The $A_{1 g}$ plasmon becomes bright. This effect is shown in Fig. 4(b) where a new absorption peak appears at $2.61 \mathrm{eV}(476 \mathrm{~nm})$ and increases in intensity with increasing ellipticity of the disk, compare Figs. 4(b) and (c). From the symmetry point of view, this change in selection rule is the same if the symmetry lowering results from having the major elliptical axis along $x$ (like in Fig. 4), along $y$, or by decreasing the size of one of the disks while keeping its circular shape. The intensity of the new bright absorption peak, however, will depend on these details.

The optical selection rules for the plasmons are important in the problem of finding PERS selection rules, because a given plasmon will only lead to a PERS resonance for optically allowed transitions. For example, the $B_{3 u}$ mode in Fig. 3(a) is excited with $x$ polarized light. Let us consider a PERS experiment where both incoming and scattered light are polarized along the dimer $x$ axis. In this configuration the incoming and scattered photons may couple to $B_{3 u}$ plasmon eigenmodes, thus

$$
\begin{gathered}
\Gamma_{\mathbf{S P}, \mathbf{P S}}^{\mathrm{coh}}[.(x, x) .]=\Gamma_{x} \otimes B_{3 u} \otimes \Gamma_{x}=B_{3 u} \\
\Gamma_{\mathbf{P S P}}^{\mathrm{coh}}[.(x, x) .]=\Gamma_{x} \otimes B_{3 u} \otimes B_{3 u} \otimes \Gamma_{x}=A_{g} .
\end{gathered}
$$

For the PSP scattering process, only the totally symmetric $A_{g}$ representation is PERS active.

Similarly to the selection rules for incoherent scattering (Section IV A), PERS enhancement is predicted to occur for a Raman-active plasmon representation in the PSP process, but for infrared- and hyper-Raman-active irreducible representations in aSP and PS processes. However, differently from the incoherent case, the selection rules are more restrictive (compare the PSP and PS,SP active irreducible representations for $D_{2 h}$ in Tables I and II). This result is indeed expected generally because in the coherent scenario the plasmonic 
TABLE II. Raman configurations in Porto notation and the PERS-active irreducible representations for the $D_{2 h}$ and $C_{\infty v}$ point groups, considering spatially coherent processes. The Porto notation lists the direction of the incoming $\mathbf{k}_{i}$ (scattered $\mathbf{k}_{s}$ ) wavevector and the incoming $\mathbf{e}_{i}$ (scattered $\mathbf{e}_{s}$ ) polarization as $\mathbf{k}_{s}\left(\mathbf{e}_{s}, \mathbf{e}_{i}\right) \mathbf{k}_{i}$. The dots in.$(\xi, \xi)$. represent any wavevector that is compatible with $\xi$ polarization. For $C_{\infty v} x$ and $y$ are interchangegable and not listed separately.

\begin{tabular}{|c|c|c|c|c|}
\hline geommetry & active plasmons & PSP & $\mathbf{S P}$ & PS \\
\hline$D_{2 h}$ & $\left(B_{1 u}, B_{2 u}, B_{3 u}\right)$ & $A_{g}$ & $\left(B_{1 u}, B_{2 u}, B_{3 u}\right)$ & \\
\hline.$(x, x)$ & $B_{3 u}$ & $A_{g}$ & $B_{3 u}$ & $B_{3 u}$ \\
\hline$\cdot(y, y)$ & $B_{2 u}$ & $A_{g}$ & $B_{2 u}$ & $B_{2 u}$ \\
\hline.$(z, z)$ & $B_{1 u}$ & $A_{g}$ & $B_{1 u}$ & $B_{1 u}$ \\
\hline.$(x, y)$ & $B_{3 u}, B_{2 u}$ & $A_{g}$ & $B_{2 u}$ & $B_{3 u}$ \\
\hline.$(y, x)$ & $B_{2 u}, B_{3 u}$ & $A_{g}$ & $B_{3 u}$ & $B_{2 u}$ \\
\hline$(x, z)$ & $B_{3 u}, B_{1 u}$ & $A_{g}$ & $B_{1 u}$ & $B_{3 u}$ \\
\hline.$(z, x)$ & $B_{1 u}, B_{3 u}$ & $A_{g}$ & $B_{3 u}$ & $B_{1 u}$ \\
\hline$\cdot(y, z)$ & $B_{2 u}, B_{1 u}$ & $A_{g}$ & $B_{1 u}$ & $B_{2 u}$ \\
\hline.$(z, y)$ & $B_{1 u}, B_{2 u}$ & $A_{g}$ & $B_{2 u}$ & $B_{1 u}$ \\
\hline$C_{\infty v}$ & $\left(\Sigma^{+}\left(A_{1}\right), \Pi\left(E_{1}\right)\right)$ & $\left(A_{1}, E_{2}, E_{4}\right)$ & $\left(A_{1}, E_{1}, E_{2}, E_{3}\right)$ & \\
\hline \multirow[t]{2}{*}{.$(x, x)$} & $\Pi$ & $\Sigma^{+}, \Delta, \Gamma$ & $\Pi, \Phi$ & $\Pi, \Phi$ \\
\hline & $E_{1}$ & $A_{1}, E_{2}, E_{4}$ & $E_{1}, E_{3}$ & $E_{1}, E_{3}$ \\
\hline \multirow[t]{2}{*}{.$(z, z)$} & $\Sigma^{+}$ & $\Sigma^{+}$ & $\Sigma^{+}$ & $\Sigma^{+}$ \\
\hline & $A_{1}$ & $A_{1}$ & $A_{1}$ & $A_{1}$ \\
\hline \multirow[t]{2}{*}{.$(x, z)$. } & $\Sigma^{+}, \Pi$ & $\Sigma^{+}, \Delta$ & $\Pi$ & $\Sigma^{+}, \Delta$ \\
\hline & $A_{1}, E_{1}$ & $A_{1}, E_{2}$ & $E_{1}$ & $A_{1}, E_{2}$ \\
\hline \multirow[t]{2}{*}{.$(z, x)$} & $\Sigma^{+}, \Pi$ & $\Sigma^{+}, \Delta$ & $\Sigma^{+}, \Delta$ & $\Pi$ \\
\hline & $A_{1}, E_{1}$ & $A_{1}, E_{2}$ & $A_{1}, E_{2}$ & $E_{1}$ \\
\hline
\end{tabular}

field symmetry is higher than in the uncorrelated incoherent scenario. Note that, for $D_{2 h}$, $\Gamma_{v e c}$ for $x, y$ and $z$ coincides with $\Gamma_{p l}$ excited for light polarized along these three directions. Still, the incoherent $\left(\Gamma^{\mathcal{H}_{p l-e l}}=\Gamma_{v e c}\right)$ and coherent $\left(\Gamma^{\mathcal{H}_{p l-e l}}=\Gamma_{p l}\right)$ scenario are different from the symmetry perspective because, in the coherent case, the incident/scattered light polar- 
ization uniquely define $\Gamma^{\mathcal{H}_{p l-e l}}$ integrally over all the space, and it does not allow summing up different $\Gamma_{v e c}$ local possibilities.

Table II lists the irreducible representations for which PERS resonances are possible (PERS-active processes) in the distinct Raman configurations for $D_{2 h}$ and $C_{\infty v}$, the later important for TERS (Section V B). Particularly interesting are crossed configurations of the incoming and scattered light $[.(x, y) . ; .(y, z) . ; .(x, z)$. etc.]. Depending on the exact PERS

process, they will selectively enhance different representations. PSP processes in crossed configurations require the resonance with two plasmon modes of different symmetry for incident and scattered lights. If the energetic separation between the plasmon eigenmodes differs strongly from the phonon energy of the sample, the resulting PERS resonances will remain weak. This is evident from Eq. (1), because only one term in the denominator vanishes, while the others remain finite. Under these conditions $\mathbf{S P}$ and $\mathbf{P S}$ processes may be of comparable or even larger intensity than PSP contributions to a PERS spectrum.

\section{PERS-ACTIVE PHONONS OF THE RAMAN PROBES}

Typically, molecules and nanostructures studied by PERS belong to a different point group than the plasmonic system. To derive the PERS selection rules for the PERS-active phonons belonging to the Raman probe, we consider the combined system of plasmonic nanostructure and sample. We will show how to obtain the enhanced phonons in a sample from the PERS-active representations of the plasmonic nanostructure they are coupled to.

As an example, we assume $D_{6 h}$ symmetry for the Raman probe. Graphene, which belongs to the $D_{6 h}$ point group, recently emerged as an interesting two-dimensional system for studying the fundamentals of SERS and TERS. ${ }^{27,40}$ It has six phonon modes belonging to the $\Gamma_{p n}=B_{2 g} \oplus E_{2 g} \oplus A_{2 u} \oplus E_{1 u}$ irreducible representations. ${ }^{42,43}$ The first-order Raman fingerprint belongs to $E_{2 g}$, which gives rise to the so-called $\mathrm{G}$ band $\left(\sim 1584 \mathrm{~cm}^{-1}\right)$ in the Raman spectra from graphene-related materials.

Another band named $\mathrm{G}^{\prime}$ or $2 \mathrm{D}\left(\sim 2700 \mathrm{~cm}^{-1}\right)$ is the second most relevant feature in the spectra of graphene-related materials, and it originates from a two-phonons second-order Raman scattering process. The symmetry for higher-order scattering involving phonons out of the Brillouin zone center $(q \neq 0)$ is derived by analysing the representations of the phonon overtone at the symmetry line/point where the phonons originate from, and then 
finding the induced representations for the entire symmetry group (at $\Gamma$ ). In general, the translational symmetry selects the allowed wavevectors to be $q_{1}=q$ and $q_{2}=-q$ for the two phonons involved, so that the second-order process obeys $q_{\text {total }}=q_{2}-q_{1}=0$. As for the non-translational symmetries, specifically for the $2 \mathrm{D}$ band, the two phonons come from the in-plane transversal optical (iTO) branch around the K point, with the highest contribution coming from the $\Gamma-K-M$ high symmetry lines, under the so-called "inner" and "outer" processes. ${ }^{44-47}$ At the $K$ point, the little group is isomorph to $D_{3 h}$, and the induced representation at $\Gamma\left(D_{6 h}\right)$ is $A_{1 g} \oplus B_{2 u}$. For regular (non-PERS) Raman scattering, the $B_{2 u}$ is not Raman active, and the hypothetical second-order scattering at this high symmetry point would happen via the totally symmetric phonon eigenmode. When moving away from the $K$ point within the $\Gamma-K-M$ line-directions, the little group changes to the $C_{2 v}$ point group, the overtone of the 2D branch (iTO like) belongs to the $A_{1}$ totally symmetric representation, with induced representation at $\Gamma$ given by $A_{1 g} \oplus E_{2 g} \oplus B_{1 u} \oplus E_{1 u}$. In regular Raman scattering (non-PERS), the $B_{1 u}$ and $E_{1 u}$ irreducible representations are not Raman active, and the 2D Raman band is composed by $A_{1 g}$ and $E_{2 g}$-like phonons, with predominance of $A_{1 g} \cdot{ }^{45}$

\section{A. Graphene on a nanodisk dimer}

When a graphene sheet is placed on a nanodisk dimer the symmetry of the combined system depends on where and how the sheet is placed with respect to the dimer. The $D_{2 h}$ symmetry of the dimer remains the symmetry of the combined system if the center of a graphene hexagon coincides with the point of inversion symmetry for the nanodimer, and the hexagon orientation is either armchair or zigzag with respect to the $x$ axis of the dimer. The SERS selection rules of $D_{6 h}$ are then found as the induced representations of the PERS-active representations of $D_{2 h}$; they are listed in the second column of Table III. The strongest SERS enhancement by a nanodisk dimer is expected when exciting the $B_{3 u}$ modes via $x$ polarized light. In this configuration the $E_{2 g}$ mode of graphene ( $\mathrm{G}$ band) is enhanced through.$(x, x)$. PSP-type scattering processes in agreement with experiment. ${ }^{40}$ The same happens for the $\mathrm{G}^{\prime}$ or $2 \mathrm{D}$ mode in graphene $\left(A_{1 g} \oplus E_{2 g}\right)$. Therefore, the two most

prominent vibrational modes observed in the Raman spectra of graphene are predicted to be enhanced in the most effective SERS configuration for a plasmonic dimer. 
TABLE III. Compatibility relations between irreducible representations belonging to the $D_{6 h}$ and $D_{2 h}$ symmetries. The first column lists the PERS-active irreducible representations of $D_{2 h}$ (compare Table I). The PERS-active phonons belonging to the irreducible representations of $D_{6 h}$ are given in the other columns, for different degrees of symmetry breaking by the combined system. See text for details. Bold-face is used to highlight the irreducible representations for phonons in the $D_{6 h}$ symmetry system $\left(\Gamma_{p n}=B_{2 g} \oplus E_{2 g} \oplus A_{2 u} \oplus E_{1 u}\right.$ plus the second-order $\left.A_{1 g}\right)$.

\begin{tabular}{|c|c|c|c|c|}
\hline \multirow[t]{2}{*}{$D_{2 h}$} & \multicolumn{4}{|c|}{ induced representations } \\
\hline & $D_{6 h}(z)$ & $D_{6 h}(x)$ & $D_{6 h}(y)$ & $D_{6 h}$ via $C_{2 v}(z)$ \\
\hline$A_{g}$ & $\mathbf{A}_{1 \mathrm{~g}}, \mathbf{E}_{2 \mathrm{~g}}$ & $\mathbf{A}_{1 \mathrm{~g}}, \mathbf{E}_{2 \mathrm{~g}}$ & $\mathbf{A}_{1 \mathrm{~g}}, \mathbf{E}_{2 \mathrm{~g}}$ & $\mathbf{A}_{1 \mathbf{g}}, \mathbf{A}_{\mathbf{2 u}}, \mathbf{E}_{\mathbf{2 g}}, E_{2 u}$ \\
\hline$A_{u}$ & $A_{1 u}, E_{2 u}$ & $A_{1 u}, E_{2 u}$ & $A_{1 u}, E_{2 u}$ & $A_{1 u}, A_{2 g}, \mathbf{E}_{2 \mathrm{~g}}, E_{2 u}$ \\
\hline$B_{1 g}$ & $A_{2 g}, \mathbf{E}_{2 \mathrm{~g}}$ & $B_{2 g}, E_{1 g}$ & $B_{1 g}, E_{1 g}$ & $A_{1 u}, A_{2 g}, \mathbf{E}_{2 \mathbf{g}}, E_{2 u}$ \\
\hline$B_{2 g}$ & $\mathbf{B}_{2 \mathrm{~g}}, E_{1 g}$ & $B_{1 g}, E_{1 g}$ & $A_{2 g}, \mathbf{E}_{2 \mathrm{~g}}$ & $B_{1 u}, \mathbf{B}_{\mathbf{2 g}}, E_{1 g}, \mathbf{E}_{1 \mathbf{u}}$ \\
\hline$B_{3 g}$ & $B_{1 g}, E_{1 g}$ & $A_{2 g}, \mathbf{E}_{2 \mathrm{~g}}$ & $\mathbf{B}_{2 \mathrm{~g}}, E_{1 g}$ & $B_{1 g}, B_{2 u}, E_{1 g}, \mathbf{E}_{\mathbf{1 u}}$ \\
\hline$B_{1 u}$ & $\mathbf{A}_{\mathbf{2 u}}, E_{2 u}$ & $B_{2 u}, \mathbf{E}_{1 \mathbf{u}}$ & $B_{1 u}, \mathbf{E}_{1 \mathbf{u}}$ & $\mathbf{A}_{1 \mathrm{~g}}, \mathbf{A}_{\mathbf{2 u}}, \mathbf{E}_{\mathbf{2 g}}, E_{2 u}$ \\
\hline$B_{2 u}$ & $B_{2 u}, \mathbf{E}_{\mathbf{1 u}}$ & $B_{1 u}, \mathbf{E}_{1 \mathbf{u}}$ & $\mathbf{A}_{\mathbf{2 u}}, E_{2 u}$ & $B_{1 g}, B_{2 u}, E_{1 g}, \mathbf{E}_{1 \mathbf{u}}$ \\
\hline$B_{3 u}$ & $B_{1 u}, \mathbf{E}_{1 \mathbf{u}}$ & $\mathbf{A}_{\mathbf{2 u}}, E_{2 u}$ & $B_{2 u}, \mathbf{E}_{1 \mathbf{u}}$ & $B_{1 u}, \mathbf{B}_{\mathbf{2 g}}, E_{1 g}, \mathbf{E}_{1 \mathbf{u}}$ \\
\hline
\end{tabular}

The selection rules are expected to change if the Raman probe is rotated. To illustrate this we list in Table III the correlation between the $D_{2 h}$ and $D_{6 h}$ point group if the $C_{6}$ axis is oriented parallel to the dimer $x$ and $y$ axis (third and fourth columns, respectively). These selection rules would apply for a molecule with $D_{6 h}$ symmetry in the center of the dimer gap. $A_{g}$ is always correlated with the totally symmetric $A_{1 g}$ and the $E_{2 g}$ representations.

The selection rules are also expected to change if the Raman probe is displaced. In the experiment reported in Ref. 40 graphene was placed on top of the nanodisk dimer that was prepared on a $\mathrm{SiO}_{2} / \mathrm{Si}$ substrate. The $D_{2 h}$ symmetry of the dimer is thereby lowered to $C_{2 v}$ for the combined system, where the $C_{2}^{z}$ axis is kept. We now have to consider the subduced representations from the symmetry lowering $\left[D_{2 h} \rightarrow C_{2 v}\left(C_{2}\right.\right.$ along $\left.\left.z\right)\right]$, followed by finding the induced representations in graphene $\left[C_{2 v} \rightarrow D_{6 h}\left(C_{2}\right.\right.$ along $\left.\left.z\right)\right]$. The result of this evaluation is listed in the last column of Table III. The modes belonging to the Raman-active $E_{2 g}$ and $A_{1 g}$ irreducible representations are again expected to be enhanced in SERS. Additionally, the graphene $A_{2 u}$ mode is activated in the.$(x, x)$. PSP scattering 
process.

Still for the $C_{2 v}\left(C_{2}\right.$ along $\left.z\right)$, interesting are also the selection rules predicted for $\mathbf{S P}$ or PS scattering events in $(x, x)$ and in crossed $(x, y)$ or $(y, x)$ polarizations. In Table II we find the $B_{3 u}$ representation to represent a PERS-active representation, which is correlated with the $B_{2 g}$ and $E_{1 u}$ representations of $D_{6 h}$ (see third column of Table III). Graphene has a $B_{2 g}$ mode around $870 \mathrm{~cm}^{-1}$ that is neither infrared nor Raman active. ${ }^{42,48}$ However, it is expected to be PERS-active considering the $D_{2 h}$ dimer.

For monolayer graphene the $A_{2 u}$ and $E_{1 u}$ phonon eigenmodes are the displacement of the entire sheet along the $z$ and $(x, y)$ directions, respectively. However, in the suspended part of graphene on dimers, these modes could exhibit a restoring force and appear at non-zero frequencies. In a graphene system with more than one layer, $A_{2 u}$ and $E_{1 u}$ modes represent the inter-layer breathing and shear modes, respectively, which appear at low frequencies (tens of $\mathrm{cm}^{-1}$ ), with values depending on the number of layers ${ }^{49}$. The $B_{2 g}$ mode of single-layer graphene splits for graphite into a silent $B_{2 g}$ mode and the infrared-active $A_{2 u}$ vibration (ZO

mode, $868 \mathrm{~cm}^{-1}$ ). Both phonon modes are expected to be enhanced in a SERS experiment with multilayer graphene, by coupling to the $B_{3 u}$ plasmons.

Further symmetry breaking is expected when the graphene location is displaced along the $x$ and $y$ direction and/or the graphene crystallographic orientation is rotated with respect to the dimer axis. Starting from $D_{2 h}$ (dimer and graphene perfectly aligned), displacements along $x$ and $y$ decrease the system symmetry to the $C_{2 v}$ point group, although each case will have a specific set of selection rule since the remaining $C_{2}$ axis varies. If graphene is displaced along two directions with respect to the dimer axes, the combined system has symmetry $C_{1 h}$ or $C_{1}$. In this case, all phonons modes become SERS active. The degree of the symmetry breaking and the resulting SERS intensity, however, is related to the field gradients felt by the phonon, within its coherence length ${ }^{29}$.

\section{B. TERS on graphene}

To study the Raman selection rules in a TERS experiment, we consider a TERS tip that is placed above a graphene sheet. The tip has a cone structure represented by the $C_{\infty v}$ point group symmetry, see Fig. 5. If the tip is placed perpendicular to the graphene plane, the symmetry of the combined system reduces to $C_{6 v}$. We proceed as outlined for graphene on 
TABLE IV. TERS selection rules for a Raman probe with $D_{6 h}$ symmetry coupled to a TERS tip belonging to the $C_{\infty v}$ point group. Notice the $\Sigma^{-}\left(A_{2}\right)$ is non-PERS-active, but we include it here for completeness. Bold-face is used to highlight the irreducible representations for phonons in the $D_{6 h}$ symmetry system $\left(\Gamma_{p n}=B_{2 g} \oplus E_{2 g} \oplus A_{2 u} \oplus E_{1 u}\right.$ plus the second-order $\left.A_{1 g}\right)$.

\begin{tabular}{ll}
\hline \hline$C_{\infty v}$ & $D_{6 h}$ via $C_{6 v}(z)$ \\
\hline$\Sigma^{+}\left(A_{1}\right)$ & $\mathbf{A}_{\mathbf{1 g}}, \mathbf{A}_{\mathbf{2 u}}$ \\
$\Sigma^{-}\left(A_{2}\right)$ non-PERS active & $A_{1 u}, A_{2 g}$ \\
$\Pi\left(E_{1}\right)$ & $E_{1 g}, \mathbf{E}_{\mathbf{1 u}}$ \\
$\Delta\left(E_{2}\right)$ & $\mathbf{E}_{\mathbf{2 g}}, E_{2 u}$ \\
$\Phi\left(E_{3}\right)$ & $B_{1 g}, B_{1 u}, \mathbf{B}_{\mathbf{2 g}}, B_{2 u}$ \\
$\Gamma\left(E_{4}\right)$ & $\mathbf{E}_{\mathbf{2 g}}, E_{2 u}$ \\
\hline \hline
\end{tabular}

a nanodimer above. The TERS-active representations of $C_{\infty v}$ are subduced onto $C_{6 v}$ and then the induced representations of $D_{6 h}$ are obtained. The resulting correlation between TERS-active representations of $C_{\infty v}$ and the representations of $D_{6 h}$ are given in Table IV.

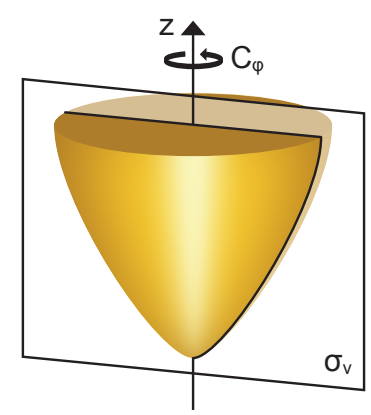

FIG. 5. (a) Schematics of the semi-infinite group $C_{\infty v}$, representative of a TERS tip structure. The symmetry elements are the identity $E$, a $C_{2}$ axis along the $z$ direction, a mirror plane $\sigma_{v}$ and an infinite possibilities of rotations $C_{\varphi}$. Some of these symmetries are shown.

According with recently published calculations, ${ }^{28}$ the TERS enhancement for the most prominent Raman modes, the $\mathrm{G}$ mode $\left(E_{2 g}\right)$ and second-order $2 \mathrm{D}$ mode $\left(A_{1 g} \oplus E_{2 g}\right)$, vanishes for linearly-polarized incident light that is propagating normal to the graphene sheet (along $z$ or the tip axis). The vanishing enhancement in this geometry results from plasmon-photon coupling, since the light polarization is in the graphene $(x, y)$ plane, whereas the tip dipole 
is along the $z$ axis. $\Gamma_{p l}^{\prime}=\Gamma_{z}$ is orthogonal to the light electric field and the transition probability is zero. ${ }^{50}$ The plasmon mode is activated by rotating the tip orientation with respect to the light propagation direction, so that $\Gamma_{p l}^{\prime}=\Gamma_{z} \oplus \Gamma_{(x, y)}$, in agreement with calculations $^{28}$. Alternatively, using radially polarized light propagating along $z$ and focused with a high numerical aperture results in an electric field with a $z$ component. ${ }^{3}$ We will now derive the TERS selection rules assuming $z$ polarized incoming and outgoing light, as utilized experimentally. ${ }^{29}$

There is a peculiar difference between spatially coherent and incoherent scattering in TERS that was already predicted from calculating near-field effects in Raman scattering and observed experimentally. ${ }^{27-29}$ The G mode $\left(E_{2 g}\right)$ was found to be enhanced in TERS for incoherent scattering, but not for coherent TERS. In contrast the 2D mode is TERS active for both coherent and incoherent scattering through its $A_{1 g}$ component. When looking at the symmetry-imposed selection rules, only the $\Sigma^{+}\left(A_{1}\right)$ representation contributes to coherent PSP-type scattering processes in the.$(z, z)$. configuration, see Table II. Group theory thus predicts $A_{1 g}$ and $A_{2 u}$ phonons to be TERS-allowed in graphene for coherent PSP processes. Incoherent scattering, on the other hand, occurs for $\Gamma_{\mathbf{P S P}}^{\operatorname{incoh}}[.(z, z)]=.\Gamma_{z} \otimes \Gamma_{\text {vec }} \otimes \Gamma_{\text {vec }} \otimes \Gamma_{z}=$ $A_{1} \oplus E_{1} \oplus E_{2}$ implying that $A_{1 g}, A_{2 u}, E_{1 u}$, and $E_{2 g}$ phonons are enhanced (see bold-faced IRs in Table IV). The symmetry analysis is in excellent agreement with the full calculation for $A_{1 g}$ and $E_{2 g}$ modes. ${ }^{27}$

SP and PS scattering processes are not allowed in the . $(z, z)$. configuration for graphene, because electron-photon coupling is only non-zero for light polarized within the plane. Inspecting the.$(x, z)$. and.$(z, x)$. configurations we find the $A_{1}, E_{1}$, and $E_{2}$ representations to be TERS-active for coherent scattering according to Table II. For incoherent scattering we reduce $\Gamma_{x, y} \otimes \Gamma_{\text {vec }} \otimes \Gamma_{z}$ and find $A_{1} \oplus E_{1} \oplus E_{2}$. Therefore, SP and PS processes enhance $A_{1 g}, A_{2 u}, E_{1 u}$, and $E_{2 g}$ phonons (see bold faced IRs in Table IV), irrespective of spatial interferences.

This interplay between processes becoming allowed and forbidden in TERS for a given scattering process and spatially coherent versus incoherent scattering makes TERS strongly dependent on the phonon coherence lengths. ${ }^{29}$ It may also result in different TERS enhancement for molecules (incoherent scattering dominates) and two-dimensional materials (interplay between coherent and incoherent scattering depending on phonon and system size). 


\section{SUMMARY}

In summary, we developed a general framework to obtain selection rules for plasmonenhanced Raman scattering. A plasmonic nanostructure has a set of PERS-active representations, which are found from its point group symmetry. The PERS-active representations for a given experimental configuration induce the PERS selection rules in a Raman probe such as a molecule, a nanostructure, or a two-dimensional solid. They are found by first considering the symmetry breaking when coupling the plasmonic system and the Raman probe (subduction) and then evaluating the induced representations in the point group of the sample, using compatibility relations.

We applied our framework to obtain the selection rules for SERS resulting from the nearfield of a plasmonic nanodisk dimer. We confirm that strongest enhancement is expected for the graphene $\mathrm{G}$ and $2 \mathrm{D}$ modes that also contribute to standard Raman scattering. Our analysis also predicts the SERS activity of $A_{2 u}, E_{1 u}$, and $B_{2 g}$ phonons, which will be interesting to study experimentally. As a second example we derived the selection rules for TERS on graphene. If the incoming and scattered light couple to the $z$ polarized tip plasmon (PSP scattering), TERS scattering for the $E_{2 g}$-symmetry G mode is forbidden in coherent, but allowed in incoherent scattering. The 2D mode is expected to show a PSPinduced TERS signal irrespective of the coherence in the scattering event. $\mathbf{S P}$ and PS processes are relevant in this two-dimensional system, and it generates enhancement in both coherent and incoherent scenario. These peculiar results generate a dependence of the TERS effect on the phonon coherence length, in excellent agreement with previous calculations and experiment. $^{27-29}$ Our analysis may readily be applied to other nanostructures and Raman probes, allowing a systematic evaluation of the SERS and TERS selection rules.

The symmetry breaking discussed in Fig. 4 provides a feeling for the effect of having nonideal plasmonic nanostructures, which are typically encountered in real experiments. In this figure the effect of losing the perfectly circular geometry for the plasmonic structures in the dimmer was discussed. Notice that, although GT is "binary", in the sense that if you break the symmetry slightly the selection rules change, in reality the effect of symmetry breaking depends on the degree of deformation. Therefore, although structures are never perfect and symmetries are broken, one may expect to have the symmetry aspects of ideal particles ruling the overall results. If deviations are much larger and make the system completely different, 
then our methodology can still be used, but the new structure has to be considering.

Furthermore, the selection rules displayed in Eqs.4(a-c) have to be generalized if one wants to address situations with enhanced degree of complexity, such as incident electric

field distribution beyond the dipole approximation, or the strong coupling regime ${ }^{51-55}$, where the intermediated states are of mixed electronic and plasmonic character. For addressing the higher-order multipole expansions, $\Gamma_{p l}^{\prime}=\Gamma_{v e c}$ should be replaced by $\Gamma_{p l}^{\prime}=\Gamma_{v e c} \oplus \Gamma_{q u a d} \oplus \ldots$ . The strong coupling regime will require setting up a hybrid system of plasmonic structure and Raman probe. In both cases, the selection rules will have to be treated case by case. The relevance of such generalizations should depend on specific experimental conditions, and should be tested experimentally. Our analysis applies to the case of standard SERS in the weak coupling regime and without extremely strong incoming field gradients. These conditions are met in most experimental SERS systems, including structures with singlemolecule sensitivity.

\section{ACKNOWLEDGEMENT}

The authors thankfully acknowledge helpful discussions with Luiz Gustavo Cançado and Sebastian Heeg. A.J. acknowledges financial support from the Humboldt Foundation. N.S.M. acknowledges financial support from Deutsche Telekom Stiftung.

1 M. Fleischmann, P. J. Hendra, and A. J. McQuillan, Journal of Photochemistry and Photobiology A: Chemistry 26, 163 (1974).

2 D. L. Jeanmaire and R. P. Van Duyne, Journal of Electroanalytical Chemistry and Interfacial Electrochemistry 84, 1 (1977).

3 L. Novotny and B. Hecht, Principles of Nano-Optics (Cambridge University Press, Cambridge, 2012).

4 E. Le Ru and P. Etchegoin, Principles of Surface-Enhanced Raman Spectroscopy and related plasmonic effects (Elsevier Science, New York, 2008).

5 S. Nie and S. Emory, Science 275, 1102 (1997). 
6 K. Kneipp, Y. Wang, H. Kneipp, L. Perelman, I. Itzkan, R. Dasari, and M. Feld, Physical Review Letters 78, 1667 (1997).

7 S. A. Maier, Plasmonics: Fundamentals and Applications (Springer: Heidelberger Platz 3, D14197 Berlin, Germany, 2007).

8 R. M. Stöckle, Y. D. Suh, V. Deckert, and R. Zenobi, Chem. Phys. Lett. 318, 131 (2000).

9 L. G. Cançado, A. Jorio, A. Ismach, E. Joselevich, A. Hartschuh, and L. Novotny, Physical Review Letters 103, 186101 (2009).

10 R. Zhang, Y. Zhang, Z. C. Dong, S. Jiang, C. Zhang, L. G. Chen, L. Zhang, Y. Liao, J. Aizpurua, Y. Luo, et al., Nature 498, 82 (2013).

11 M. Moskovits, Phys Chem Chem Phys 15, 5301 (2013).

12 V. M. Shalaev and A. K. Sarychev, Phys. Rev. B 57, 13265 (1998).

13 J. R. Lombardi and R. L. Birke, The J. Phys. Chem. C 112, 5605 (2008).

14 Y. Luo, A. Aubry, and J. B. Pendry, Phys. Rev. B 83, 155422 (2011).

15 M. Finazzi and F. Ciccacci, Phys. Rev. B 86, 035428 (2012).

16 N. S. Mueller, S. Heeg, and S. Reich, Phys. Rev. A 94, 023813/1 (2016).

17 P. Roelli, C. Galland, N. Piro, and T. J. Kippenberg, Nat. Nanotec. 11, 164 (2015).

18 M. K. Schmidt, R. Esteban, A. González-Tudela, G. Giedke, and J. Aizpurua, ACS Nano 10, $6291(2016)$.

19 T. Inui, Y. Tanabe, and Y. Onedera, Group Theory and its Application in Physics (Springer, New York, 1996), 2nd ed.

20 E. B. Wilson, J. C. Decius, and P. C. Cross, Molecular Vibrations (Dover, 2001).

21 M. S. Dresselhaus, G. Dresselhaus, and A. Jorio, Group Theory: Application to the Physics of Condenced Matter (Springer, Berlin Heidelberg, 2008).

22 M. Cardona, in Light Scattering in Solids II (Springer, New York, 1982).

23 L. Chuntonov and G. Haran, Nano Lett. 11, 2440 (2011).

24 W. Zhang, B. Gallinet, and O. J. F. Martin, Phys. Rev. B 81, 233407 (2010).

25 D. W. Brandl, N. A. Mirin, and P. Nordlander, J. Phys. Chem. B 110, 12302 (2006).

26 J. R. Lombardi and R. L. Birke, The J. Chem. Phys. 136, 1444704 (2012).

27 L. G. C. cado, R. Beams, A. Jorio, and L. Novotny, Phys. Rev. X 4, 031054 (2014).

28 R. V. Maximiano, R. Beams, L. Novotny, A. Jorio, and L. G. Cançado, Physical Review B 85, 235434 (2012). 
29 R. Beams, L. G. Cancado, S.-H. Oh, A. Jorio, and L. Novotny, Phys. Rev. B 113, 186101 (2014).

30 S. Heeg, A. Oikonomou, R. Fernández-García, C. Lehmann, S. A. Maier, A. Vijayaraghavan, and S. Reich, Nano Letters 14, 1762 (2014).

31 M. I. Aroyo, J. M. Perez-Mato, C. Capillas, E. Kroumova, S. Ivantchev, G. Madariaga, A. Kirov, and H. Wondratschek, Z. Krist. 221, 15 (2006).

32 M. I. Aroyo, A. Kirov, C. Capillas, J. M. Perez-Mato, and H. Wondratschek, Acta Cryst A62, $115(2006)$.

33 Available online at http://www.gernot-katzers-spice-pages.com/character_tables/index.html, accessed 11/18/2016.

34 U. Hohenester and A. Trügler, C. Phys. Comm. 183, 370 (2012).

35 F. J. G. de Abajo and A. Howie, Phys. Rev. B 65, 115418 (2002).

36 D. L. Rousseau, R. P. Bauman, and S. P. S. Porto, J. Raman Spec. 10, 253 (1981).

37 C. Awada, J. Plathier, C. Dab, F. Charra, L. Douillard, and A. Ruediger, Phys. Chem. Chem. Phys. 18, 9405 (2016).

38 P. Kusch, S. Matel, N. M. Azpiazu, S. Heeg, R. Gobrachev, F. Schedin, U. Hübner, J. I. Pascual, S. Reich, and R. Hillenbrand (2016), submitted.

39 F. Schedin, E. Lidorikis, A. Lombardo, V. G. Kravets, A. K. Geim, A. N. Grigorenko, K. S. Novoselov, and A. C. Ferrari, ACS Nano 4, 5617 (2010).

40 S. Heeg, R. Fernández-García, A. Oikonomou, F. Schedin, R. Narula, S. A. Maier, A. Vijayaraghavan, and S. Reich, Nano Letters 13, 301 (2013).

41 S. Dodson, M. Haggui, R. Bachelot, J. Plain, S. Li, and Q. Xiong, The Journal of Physical Chemistry Letters 4, 496 (2013).

42 S. Reich and C. Thomsen, Phil. Trans. A 362, 2271 (2004).

43 L. M. Malard, M. H. D. G. aes, D. L. Mafra, M. S. C. Mazzoni, and A. Jorio, Phys. Rev. B 79, $125426(2009)$.

44 A. Grüneis, R. Saito, G. G. Samsonidze, T. Kimura, M. Pimenta, A. Jorio, A. Souza Filho, G. Dresselhaus, and M. Dresselhaus, Physical Review B 67, 165402 (2003).

45 D. Yoon, H. Moon, Y.-W. Son, G. Samsonidze, B. H. Park, J. B. Kim, Y. Lee, and H. Cheong, Nano letters 8, 4270 (2008).

46 M. Mohr, J. Maultzsch, and C. Thomsen, Physical Review B 82, 201409 (2010). 
47 D. Mafra, E. Moujaes, S. Doorn, H. Htoon, R. Nunes, and M. Pimenta, Carbon 49, 1511 (2011).

48 M. Mohr et al., Phys. Rev. B 76, 035439 (2007).

49 D. Boschetto, L. Malard, C. H. Lui, K. F. Mak, Z. Li, H. Yan, and T. F. Heinz, Nano Lett. 14, $4620(2014)$.

50 In evaluating TERS selection rules, one has to consider that only the near field of the $z$ polarized $A_{1}\left(\Sigma^{+}\right)$plasmon overlaps spatially with the graphene monolayer, not the $(x, y)$ polarized $E_{1}(\Pi)$ plasmon that is too far away. Consequently, only excitation of the $z$ polarized plasmon contributes to TERS.

51 K. Lopata and D. Neuhauser, J. Chem. Phys. 130, 104707 (2009).

52 K. Lopata and D. Neuhauser, J. Chem. Phys. 131, 014701 (2009).

53 A. Manjavacas, F. J. García de Abajo, and P. Nordlander, Nano Lett. 11, 23182323 (2011).

54 M. Sukharev and A. Nitzan, Phys. Rev. A 84, 043802 (2011).

55 A. J. White, M. Sukharev, and M. Galperin, Phys. Rev. B 86, 205324 (2012). 